\title{
APPLICATION OF A MERIT FUNCTION BASED INTERIOR POINT METHOD TO LINEAR MODEL PREDICTIVE CONTROL
}

\author{
Prashant Bansode ${ }^{1}$, D. N. Sonawane ${ }^{2}$ and Prashant Basargi ${ }^{3}$ \\ ${ }^{1,2,3}$ Department of Instrumentation and Control Engineering, College of Engineering, \\ Pune, Maharashtra
}

\begin{abstract}
This paper presents robust linear model predictive control (MPC) technique for small scale linear MPC problems. The quadratic programming $(Q P)$ problem arising in linear MPC is solved using primal dual interior point method. We present a merit function based on a path following strategy to calculate the step length $\alpha$, which forces the convergence of feasible iterates. The algorithm globally converges to the optimal solution of the QP problem while strictly following the inequality constraints. The linear system in the $Q P$ problem is solved using $L D L^{T}$ factorization based linear solver which reduces the computational cost of linear system to a certain extent. We implement this method for a linear MPC problem of undamped oscillator. With the help of a Kalman filter observer, we show that the MPC design is robust to the external disturbances and integrated white noise.
\end{abstract}

\section{KEYWORDS}

Model Predictive Control, Quadratic Programming, Primal Dual Interior Point Methods, Merit Function

\section{INTRODUCTION}

MPC is an advanced control strategy. It predicts the effect of the input control signal on internal states and the output of the plant. At each sampling interval of this strategy, the plant output is measured, the current state of the plant is estimated and based on these calculations a new control signal is delivered to the plant. The purpose of the new control input is to ensure that the output signal tracks the reference signal while satisfying the objective function of the MPC problem without violating the given constraints, see [1]-[3]. The objective function is defined in such a way that the output signal tracks the reference signal while it eliminates the effect of known disturbances and noise signals to achieve closed loop control of the plant. The constraints can be given in terms of bounds on input and output signals. In reality, these constraints can be the physical limitations on actuator movements, often called as hard constraints. MPC strategy handles physical constraints effectively which makes it suitable for industrial applications.

MPC problem can be formulated as a quadratic programming (QP) optimal control problem, see [2]-[4]. This QP problem is solved at a specific sampling interval to compute a sequence of current and future optimal control inputs from the predictions made on the current state and the plant output over a finite horizon known as prediction horizon; see [4]. Only the current optimal input is implemented as the plant input and the plant is updated for internal states and the plant output. Again, at next sampling interval the updated plant information is used to formulate a new

DOI : 10.5121/ijitmc.2014.2205 
optimal control problem and the process is repeated. MPC problem may require a long sampling time depending upon computational complexities associated with the QP problem solving algorithm. Therefore, the application of MPC is restricted to systems with slow dynamic performance; such applications are found in chemical industries. Interior point methods are widely accepted as the QP problem solving techniques in MPC applications. In last two decades, many research literatures have discussed the application of interior point methods to MPC problems with relatively faster dynamics by exploiting the structure of QP problems arising in MPC, see [1], [5], [6], [11]. The general discussion on interior point methods is seen in [1], [5], [6], [7], [8], [9], [10], [11]. For discussion on MPC as an optimal control strategy, see [2], [3], [4], [12], [13]. For small scale MPC problems (with state dimension not more than five) we need not exploit the structure of the problem, rather the effort should be in the direction to render faster execution of QP solving techniques by introducing new step length strategies and improving linear solvers while retaining the stability of the system, see [10], [11].

If we consider the barrier method (earlier form of interior point method) for MPC problems, it has computational complexities associated with calculating the inverse of the Hessian matrix [9]. The computational cost of inverting the Hessian is $O\left(n^{3}\right)$, Secondly, the barrier method requires two distinct iterations to update primal and dual variables, see [9]. Moreover, this method works only for strictly feasible problems. If barrier methods are considered for MPC problems, the above issues will dramatically affect the computational time of a numerical QP algorithm, as a result, also the sampling time of the MPC. The primal dual interior point method has several advantages over barrier methods such as updates of primal and dual variables are computed in a single iteration, efficiency in terms of accuracy and ability to work even when problem is not strictly feasible, and inverting the Hessian matrix is not required. Hence, it is more cost effective to select the primal dual interior point methods for QP problems than selecting the barrier methods. Further, faster convergence of iterates can be achieved by considering new step length strategies in the primal dual algorithm. One of such strategies is to measure progress to the solution by monitoring a merit function. We can measure progress to the solution between two successive iterates using a proper merit function, see [15]-[17]. We consider a logarithmic merit function that contains all the possible information required to minimize a convex quadratic objective function.

This paper discusses the primal dual interior point method to solve linear model predictive control problems with convex quadratic objective function and linear inequality constraints on the control input. The proposed method utilizes a log barrier penalty function as a merit function which estimates the progress to the solution and forces convergence of the primal dual feasible iterates, hence making the algorithm to execute faster while strictly maintaining the feasibility. To solve a linear system for computation of Newton steps, we use $\mathrm{LDL}^{\mathrm{T}}$ factorization linear solver which reduces the computational cost of the linear solver from $O\left(n^{3}\right)$ to its $O\left((1 / 3) n^{3}\right)$, see [9]. The step length selection is based on the mathematical condition derived using the merit function, and only that step length value is selected for which a sufficient decrease in the derived condition is observed. Finally, we implement this algorithm to a problem of undamped oscillator described in [3] using MATLAB platform. In results, we show that the proposed method solves a MPC problem within the specified sampling interval. Secondly, using Kalman filter observer we also prove that our MPC design is robust in terms of disturbance and noise rejection.

We organize the paper as follows: Section 2 describes linear MPC plant model and its QP formulation. In section 3, we discuss the proposed QP solving algorithm; section 4 includes MPC implementation on the undamped oscillator problem with simulation results. Section 5 concludes our paper. 


\section{MPC PROBLEM FORMULATION}

The linear MPC comprises a linear plant model, convex quadratic objective function and linear inequality constraints. The plant model is described in the section below.

\subsection{Plant Model}

We assume a state space model of the plant as given below:

$$
\begin{aligned}
& x_{t+1}=A x_{t}+B u_{t}+G w_{t}, \\
& y_{t}=C x_{t}+D u_{t}+v_{t} .
\end{aligned}
$$

Where $x_{t} \in R^{n_{x}}, u_{t} \in R^{n_{u}}, y_{t} \in R^{n_{y}}$.

Further, we assume that the plant is subjected to white noise disturbances i.e. unbiased process noise $w_{t}$ and measurement noise $v_{t}$ which are Gaussian distributed with zero mean. We design a Kalman filter observer to calculate the estimates of the current state and the plant output. If $N_{p}$ is a prediction horizon, MPC computes these estimates over the entire prediction horizon from time $t+l$ until time $t+N_{p}$ based on the information about previous plant measurements available from $t$ 1 up to current time $t$. Let us represent the optimal estimates of the state space equations as given below:

$$
\begin{aligned}
& \bar{x}_{t+i+1}=A \bar{x}_{t+i}+B u_{t+i}+G w_{t+i}, \\
& \bar{y}_{t+i}=C \bar{x}_{t+i}+D u_{t+i}+v_{t+i} .
\end{aligned}
$$

Where, $i=1, \ldots, N_{p}$.

\subsection{Control Objective}

The main objective of MPC is to force $y_{t}$ to track the reference signal, denoted by $r_{t}$ while rejecting the disturbance signal, denoted by $d_{t}$. A control objective can be represented mathematically using an objective function which obeys certain inequality constraints. By including a penalty term such as $\left\|y_{t}-r_{t}\right\|$ in the objective function, we penalize deviations of the output from the reference. Secondly by adding a term like $\left\|u_{t}-u_{t-1}\right\|$ to the objective functions we penalize the control signal $u_{t}$ not to exceed a given limit, say $l b \leq u_{t} \leq u b$, where $l b$ and $u b$ are lower and upper bounds on the input respectively. The objective function for the MPC problem is defined below as:

$$
J=\frac{1}{2} \sum_{k=1}^{P}\left\|\bar{y}_{t+k}-r_{t+k}\right\|_{Q}^{2}+\left\|u_{t+k}-u_{t+k-1}\right\|_{S}^{2} .
$$

In the above, matrices $Q$ and $S$ act as weight factors on $y_{t}$ and $u_{t}$ respectively, and they are assumed to be symmetric and positive definite. Further, we can illustrate that:

$\left\|\bar{y}_{t+k}-r_{t+k}\right\|_{Q}^{2}=\left(\bar{y}_{t+k}-r_{t+k}\right)^{T} Q\left(\bar{y}_{t+k}-r_{t+k}\right)$. 
And

$\left\|u_{t+k}-u_{t+k-1}\right\|_{S}^{2}=\left(u_{t+k}-u_{t+k-1}\right)^{T} S\left(u_{t+k}-u_{t+k-1}\right)$.

We derive the objective function in a compact form as given below:

$J=\frac{1}{2} U_{t}^{T} H U_{t}+h^{T} U_{t}$.

Where $U_{t}$ is the optimal control input as a solution to the above QP problem. The state space matrices and weighting factors do not change unless modified by the user. Hence, the matrix $H$ can be computed prior to the plant simulation i.e. it is computed offline. The basic formulation of a MPC problem as a QP optimal control problem is cited in [2]-[4].

\subsection{Inequality Constraints}

Inequality constraints on the control input signal prevent it from exceeding a specific limit. We describe the inequality constraints on the control input as given below:

$U^{\min }<U<U^{\max }$ or

$\left[\begin{array}{c}-I \\ I\end{array}\right] U=\left[\begin{array}{c}-U^{\min } \\ U^{\max }\end{array}\right]$

With the control objective and the inequality constraints formulated as shown in (6) and (7) respectively, we compute the optimal control input $U_{t}^{*}$ as a solution to QP optimal control problem shown below:

$U_{t}^{*}=\min J$

s.t $P_{i n} U_{t} \leq q_{\text {in }}$.

\section{QP SOLVER}

In this section, we discuss the algorithm for primal dual interior point method. At first, we define Lagrangian function and derive its K-K-T optimality conditions. In later part, we discuss the merit function and finally the algorithm.

Consider an inequality constrained QP problem in general form as given below:

$\min J=\frac{1}{2} u^{T} H u+h^{T} u$,

subject to $P u \leq q$.

For the sake of simplicity of the algorithm, we use notations $u, P$ and $q$ for $U_{t}, P_{\text {in }}$ and $q_{\text {in }}$ respectively. 


\subsection{K-K-T Optimality Conditions}

The Lagrangian of the above QP problem is given below:

$L(u, \lambda)=u^{T} H u+h^{T} u+\lambda^{T}(P u-q)$.

Where, $\lambda$ is the Lagrange multiplier for the inequality constraints and $s$ is the slack variable associated with it. The optimality conditions for the general QP in (9) with $u$ as a primal variable and $\lambda$ as a dual variable are given below:

$$
\begin{aligned}
& H u+P^{T} \lambda=-h, \\
& P u+s=q, \\
& \lambda^{T} s=0, \\
& \lambda, s>0 .
\end{aligned}
$$

Since we consider MPC-QP problems, they are assumed to be convex in nature and $H$ is assumed to be positive symmetric semidefinite matrix. Hence, the Optimality conditions derived above are both necessary and sufficient.

The augmented linear system for above optimality conditions is given by:

$$
\left[\begin{array}{cc}
H & -P \\
P^{T} & \lambda^{-1} s
\end{array}\right]\left[\begin{array}{l}
\Delta u \\
\Delta \lambda
\end{array}\right]=-\left[\begin{array}{l}
r_{d} \\
r_{p}
\end{array}\right] .
$$

Where $r_{d}$ and $r_{p}$ are the residues as given below:

$r_{d}=H u+P^{T} \lambda+h$ and $r_{p}=P u+s-q$.

\subsection{Merit Function}

We consider a force field interpretation theory [3] for selection of a proper merit function. We consider force field acting on a particle in a feasible region as given below:

$$
F_{i}(u)=-\nabla\left(-\log \left(-f_{i}(u)\right)\right)=\frac{\nabla f_{i}(u)}{f_{i}(u)} .
$$

The force $F_{i}(u)$ is associated with each constraint acting on a particle when it is at position $u$. The potential associated with the total force field generated by constraints is summation of all such force fields which is given as the logarithmic barrier function $\phi$. As the particle moves toward the boundary of a feasible set, the bound on the particle grows strong repelling it away from the boundary. 
International Journal of Information Technology, Modeling and Computing (IJITMC) Vol. 2, No.2, May 2014

The merit function we considered depends essentially upon a log barrier function given by:

$f=\mu \sum_{i=1}^{m} \log \left(\lambda^{T}\left(p_{i}^{T} u-q_{i}\right)\right)$, with $\operatorname{dom} \phi=\left\{u \in R^{m} \mid f_{i}(u)<0, i=1, \ldots, m\right\}$.

Where $m=$ number of inequality constraints.

Now, if we penalise the Lagrangian $L$ with the $\log$ barrier function $f$, we get:

$\phi_{\mu}(w)=L(u, \lambda)-\mu \sum_{i=1}^{m} \log \left(\lambda^{T}\left(p_{i}^{T} u-q_{i}\right)\right)$.

Substituting for $L(u, \lambda)$ in (15), we get:

$\phi_{\mu}(w)=J+\lambda^{T}(P u-q)-\mu \sum_{i=1}^{m} \log \left(\lambda^{T}\left(p_{i}^{T} u-q_{i}\right)\right)$.

Where, $w=(u, \lambda)$ which satisfies $(u, \lambda)>0$.

The merit function $\phi_{\mu}(w)$ can be thought of as another QP minimization problem because $f$ is convex and strictly satisfy the constraints, it is given below as:

$\min \phi_{\mu}(w)$,

$P u \leq q$.

The problem in (17) decreases along the direction $\Delta w$ forcing the convergence of $w$ towards the solution of (12) which is unique, say $w^{*}$. We can say that:

$\phi_{\mu}\left(w_{\mu}^{i+1}\right) \leq \phi_{\mu}\left(w_{\mu}^{i}\right)$

We derive the conditions for a stationary point $w^{*}$ by computing $\nabla \phi_{\mu}(w)=0$ using its directional gradient $\nabla \phi_{\mu}(w)$ as given below:

$\nabla \phi_{\mu}(w)=\nabla_{u} \phi_{\mu}(w)+\nabla_{\lambda} \phi_{\mu}(w)$,

$\nabla_{u} \phi_{\mu}(w)=\nabla_{u} J+P \lambda-\mu \sum_{i=1}^{m} \frac{p_{i}}{\left(p_{i}^{T} u-q_{i}\right)}$,

$\nabla_{\lambda} \phi_{\mu}(w)=P u-q-\mu \sum_{i=1}^{m} \frac{1}{\lambda}$. 
Further, we can modify (20) and (21) as:

$$
\begin{aligned}
& \nabla_{u} \phi_{\mu}(w)=\nabla_{u} J+\nabla_{u}(P u-q) \lambda-\mu \sum_{i=1}^{m} \nabla\left(P_{i} u-q_{i}\right)\left(p_{i}^{T} u-q_{i}\right)^{-1} . \\
& \nabla_{\lambda} \phi_{\mu}(w)=P u-q-\mu \sum_{i=1}^{m} \Lambda^{-1} e .
\end{aligned}
$$

In equations (22) and (23), $\nabla_{u} \phi_{\mu}(w)$ and $\nabla_{\lambda} \phi_{\mu}(w)$ act as force fields on particles at position $u$ and $\lambda$ respectively, forcing them away from the boundaries of the convex region to follow the central path defined by the set of points $\left(u^{*}, \lambda^{*}\right)$, for $u>0$ and $\lambda>0$.

Where $\Lambda=$ Diagonal $\left(\lambda_{i}, \ldots, \lambda_{m}\right)$ and $e$ is the unit vector associated with $\Lambda$.

The directional gradient $\nabla \phi_{\mu}(w)$ at point $w$ is nonpositive, to assure that $\phi_{\mu}(w)$ is only decreasing as $w$ moves along the central path toward the optimal solution $w^{*}$. Now, the progress to the QP solution can be monitored using the Newton step $\Delta w$, associated step length $\alpha$ and a point $w=w+\alpha \Delta w$. The choice of sufficient decrease condition is in spirits with the sufficient decrease condition mentioned in [9], [12]. We consider the Taylor's series expansion of $\phi_{\mu}(w+\alpha \Delta w)$ at a point $w$ which is given as:

$\phi_{\mu}(w+\alpha \Delta w) \leq \phi_{\mu}(w)+\alpha \nabla \phi_{\mu}(w)^{T} \Delta w$.

The above expansion term satisfies the condition given in (18); it also shows that $\phi_{\mu}(w)$ reduces as $w$ moves towards its optimal solution. The backtracking search algorithm is used to compute $\alpha$ such that $w+\alpha \Delta w \geq 0$. We stop the search algorithm if sufficient decrease in the condition given in (24) is satisfied, if sufficient decrease in (24) is not observed then the value of $\alpha$ is decreased and set to a value using $\alpha=\alpha^{*} \beta$ where $\beta \in(0,1)$ and the process is repeated until the sufficient decrease condition is satisfied. Hence, only those values of $\alpha$ are chosen for which algorithm generates feasible iterates and values of $\alpha$ for which algorithm deviates from the central path are rejected.

\subsection{Algorithm}

Let $w_{0}=\left(u_{0}, \lambda_{0}\right)$ be an initial point satisfying $\left(u_{0}, \lambda_{0}\right)>0$ and assume that $H_{0}$ is available for $k=0$, $1, \ldots, \mu>1, \varepsilon_{\text {feas }}>0, \varepsilon>0$.

Do

1. Choose $\sigma_{k} \in(0,1)$ and set $\mu_{k}=\sigma_{k} *$ duality gap.

Where duality gap $=\lambda_{k}^{T} s_{k} / m$ and $m=$ number of inequality constraints.

2. Compute Newton steps $\Delta u_{k} \& \Delta \lambda_{k}$ by solving following augmented linear system. As proposed, we solve the linear system using $L D L^{T}$ factorization method.

$$
\left[\begin{array}{cc}
H_{k} & -P_{k} \\
P_{k}^{T} & \lambda_{k}^{-1} s_{k}
\end{array}\right]\left[\begin{array}{l}
\Delta u_{k} \\
\Delta \lambda_{k}
\end{array}\right]=-\left[\begin{array}{l}
r_{d k} \\
r_{p_{k}}
\end{array}\right],
$$


and $\nabla s_{k}=\lambda^{-1}\left(r_{s}-S \Delta \lambda\right)$.

Where $r_{d}=H u+P^{T} \lambda+h, r_{p}=P u+s-q-\lambda S e$ and $r_{s}=S \Lambda e$

And $S=$ Diagonal $\left(s_{i}, \ldots, s_{m}\right)$.

3. Compute a step size $\alpha$ by using a backtracking line search.

Set $\alpha=\alpha_{\text {max }}=1$,

and test the sufficient decrease condition: $\phi_{\mu}(w+\alpha \Delta w) \leq \phi_{\mu}(w)+\alpha \nabla \phi_{\mu}(w)^{T} \Delta w$,

Where $\alpha \in(0,1)$.

If the test function is not decreased sufficiently, decrease and compute $\alpha$ as given below:

$\alpha=\alpha_{\max } \min \left\{1, \beta \min \left\langle-\frac{\lambda}{\Delta \lambda} \mid \Delta \lambda<0\right\rangle\right\}$,

Where $\beta \in(0,1)$.

4. Update $w_{k+1}=w_{k}+\alpha_{k} \Delta w_{k}$.

Until $\left\|r_{\text {pri }}\right\|_{2} \leq \varepsilon_{\text {feas }},\left\|r_{\text {dual }}\right\|_{2} \leq \varepsilon_{\text {feas }}$, where $\varepsilon_{\text {feas }}=$ tolerance level.

\subsection{LDL $^{\mathrm{T}}$ Factorization}

The linear system given in (12) is of the form $A X=B$, where $A$ is a Hermitian positive definite matrix, the algorithm uniquely factors $A$ as:

$$
A=L D L^{T}\left(\operatorname{Cost} O\left((1 / 3) n^{3}\right)\right)
$$

$L$ is a lower triangular square matrix with unity elements at diagonal positions, $D$ is the diagonal matrix, and $L^{T}$ is the Hermitian transpose of $L$.

The equation $L D L^{T} X=B$ is solved for $X$ by the following steps.

1. Substitute

$$
Y=D L^{T} X
$$

2. Substitute

$$
Z=L^{T} X
$$

3. Solve

$L Y=B$, using forward substitution $\left(\operatorname{Cost} O\left(n^{2}\right)\right)$

$D Z=Y$, solving diagonally $($ Cost $O(n))$

$L^{T} X=Z$, using backward substitution $\left(\operatorname{Cost} O\left(n^{2}\right)\right)$

The overall cost of the above linear solver is dominated by cost of $A=L D L^{T}$ which is $O\left((1 / 3) n^{3}\right)$. 


\section{MPC IMPLEMENTATION}

\subsection{Plant Model}

We consider a following problem of undamped oscillator for implementing linear MPC strategy; this problem is cited from [3]. Its state space representation is given below:

$$
\begin{aligned}
& {\left[\begin{array}{l}
\dot{x}_{1}(t) \\
\dot{x}_{2}(t)
\end{array}\right]=\left[\begin{array}{cc}
0 & 1 \\
-4 & 0
\end{array}\right]\left[\begin{array}{l}
x_{1}(t) \\
x_{2}(t)
\end{array}\right]+\left[\begin{array}{l}
1 \\
0
\end{array}\right] u(t),} \\
& y(t)=\left[\begin{array}{ll}
0 & 1
\end{array}\right]\left[\begin{array}{l}
x_{1}(t) \\
x_{2}(t)
\end{array}\right]
\end{aligned}
$$

The state space model given in (25) is both controllable and observable. The discrete time state space representation of the system in (25) with sampling time of 0.1 seconds is given below

$$
\begin{aligned}
& {\left[\begin{array}{l}
x_{1}(k+1) \\
x_{2}(k+1)
\end{array}\right]=\left[\begin{array}{cc}
0.9801 & 0.0993 \\
-0.3973 & 0.9801
\end{array}\right]\left[\begin{array}{l}
x_{1}(k) \\
x_{2}(k)
\end{array}\right]+\left[\begin{array}{c}
0.0993 \\
-0.0199
\end{array}\right] u(k),} \\
& y(k)=\left[\begin{array}{ll}
0 & 1
\end{array}\right]\left[\begin{array}{l}
x_{1}(k) \\
x_{2}(k)
\end{array}\right] .
\end{aligned}
$$

The control signal $u(k)$ has inequality constraints given as $-25 \leq u(k) \leq 25$. We set the prediction horizon to $N_{p}=10$ and the control horizon to $N_{c}=5$ with the MPC sampling interval of $T_{s}=1$ unit.

In our case, we introduce an integrated white noise signal in the plant which is assumed to be Gaussian distributed with zero mean and covariance matrices $Q_{o}$ and $R_{o}$ respectively.

The new plant model is given as:

$$
\begin{aligned}
& {\left[\begin{array}{l}
x(k+1) \\
d(k+1)
\end{array}\right]=\left[\begin{array}{ll}
A & 0 \\
0 & I
\end{array}\right]\left[\begin{array}{c}
x(k) \\
d(k)
\end{array}\right]+\left[\begin{array}{l}
B \\
0
\end{array}\right] u(k),} \\
& y(k)=\left[\begin{array}{ll}
C & I
\end{array}\right]\left[\begin{array}{c}
x(k) \\
d(k)
\end{array}\right]
\end{aligned}
$$

\subsection{Observer Design}

The observer equations for the state space representation in (27) are given as:

$$
\left[\begin{array}{l}
\bar{x}(k+1) \\
\bar{d}(k+1)
\end{array}\right]=\left(\left[\begin{array}{cc}
A & 0 \\
0 & I
\end{array}\right]-\left[\begin{array}{c}
L_{x} \\
L_{d}
\end{array}\right]\left[\begin{array}{ll}
C & I
\end{array}\right]\right)\left[\begin{array}{c}
\bar{x}(k+1) \\
\bar{d}(k+1)
\end{array}\right]+\left[\begin{array}{l}
B \\
0
\end{array}\right] u(k)+\left[\begin{array}{c}
L_{x} \\
L_{d}
\end{array}\right] y(k) \text {. }
$$

For the plant subjected to white noise disturbances, we design Kalman filter observer with the gain matrix $L$ such that mean value of the sum of estimation errors is minimum. We obtain $L$ recursively using MATLAB command dlqe, see [3], [4] which is given as:

$$
L=\operatorname{dlqe}\left(A_{o}, G_{o}, C_{o}, Q_{o}, R_{o}\right) \text {. }
$$


With $A_{o}=A, G_{o}=[I]_{2 \times 2}, C_{o}=\left[\begin{array}{ll}1 & 1\end{array}\right], Q_{o}=[I]_{2 \times 2}$ and $R_{o}=1$, we obtain $L=\left[\begin{array}{c}-0.43 \\ 0.52\end{array}\right]$.

For the state space representation in (26), we obtain matrices $Q, R, H$ and $h$ as given below:

$$
Q=[I]_{2 \times 2}, R=\left[\begin{array}{l}
0.2 \\
0.1
\end{array}\right], H=\left[\begin{array}{lllll}
0.2022 & 0.1785 & 0.1498 & 0.1180 & 0.0860 \\
0.1785 & 0.1629 & 0.1391 & 0.1117 & 0.0828 \\
0.1498 & 0.1391 & 0.1233 & 0.1008 & 0.0763 \\
0.1180 & 0.1117 & 0.1008 & 0.0763 & 0.0666 \\
0.0563 & 0.0551 & 0.0557 & 0.0462 & 0.0387
\end{array}\right] \text { and } h=\left[\begin{array}{l}
0.8461 \\
0.7845 \\
0.6922 \\
0.5758 \\
0.4450
\end{array}\right] \text {. }
$$

\section{SIMULATION RESULTS}

For the problem mentioned in (26), the simulations are run for 150 sampling intervals with $T_{s}=1$, output disturbance is introduced for samples with $t>50$. The results shown in figure 1 are the plant states $x_{1}$ and $x_{2}$ and their state estimations. Figure 2 shows the output response $y_{t}$ to the plant in the presence of white noise and the output disturbances. In figure 3, we show the plant output response $y_{t}$ without the presence of the white noise and output disturbances. We testify the proposed method by comparing it with Matlab's standard QP solver 'Quadprog' for the accuracy of computed values of $u_{t}$. In figure 4, we plot the graph for computational accuracy as well as time comparison between the proposed method and Quadprog tool. In the graphs below, we use PD-IP as a legend for proposed interior point method.
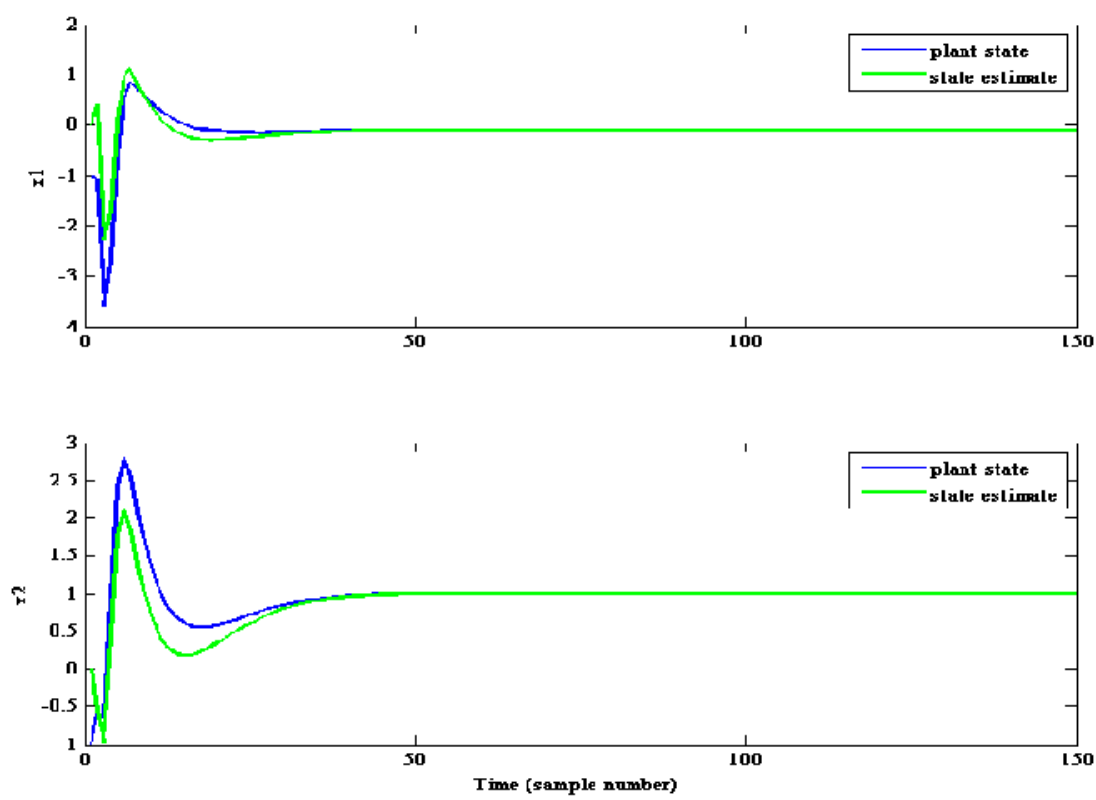

Figure 1. Plant states and their estimation using Kalman filter 
International Journal of Information Technology, Modeling and Computing (IJITMC) Vol. 2, No.2, May 2014
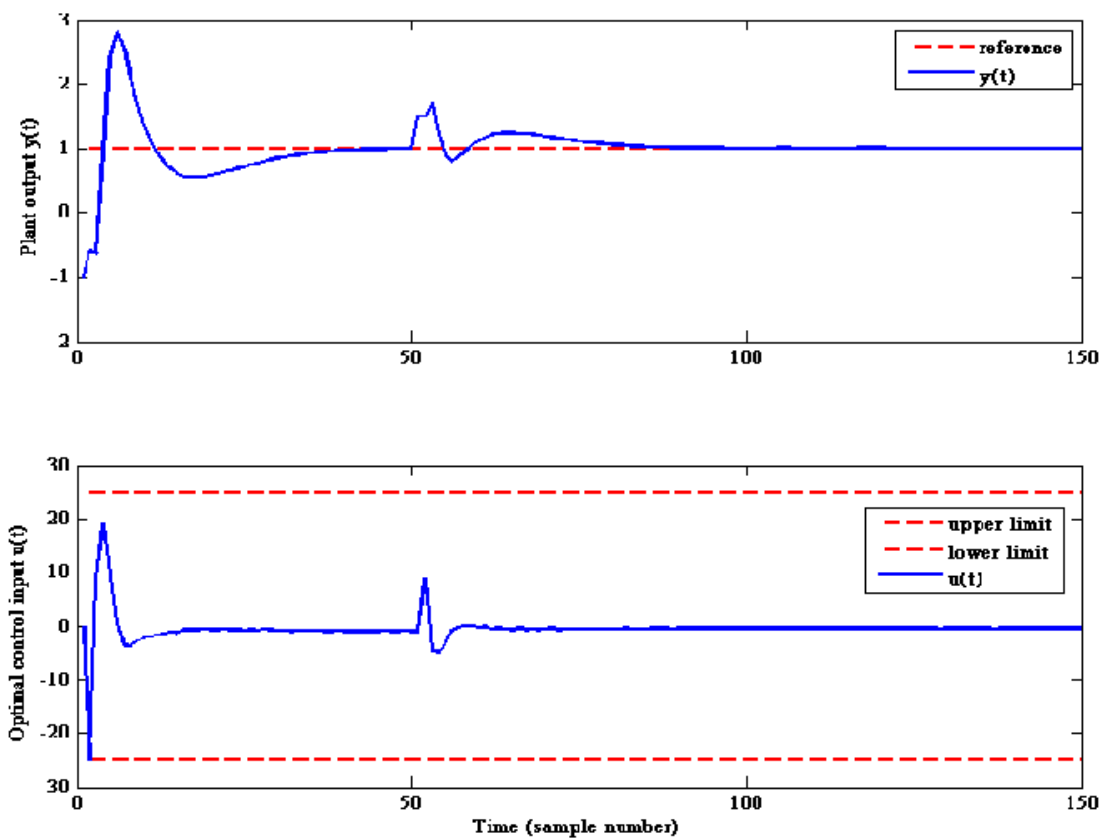

Figure 2. Plant output with disturbances and integrated white noise
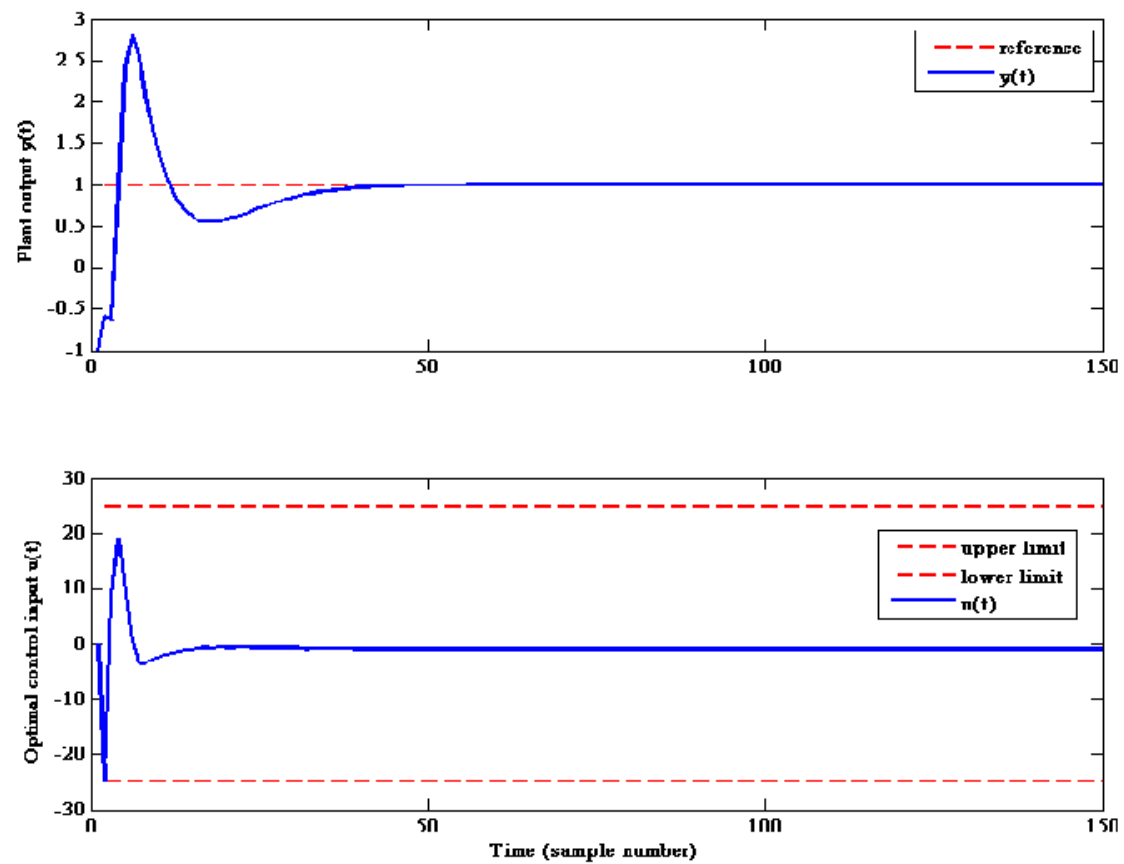

Figure 3. Plant output without disturbances and integrated white noise 

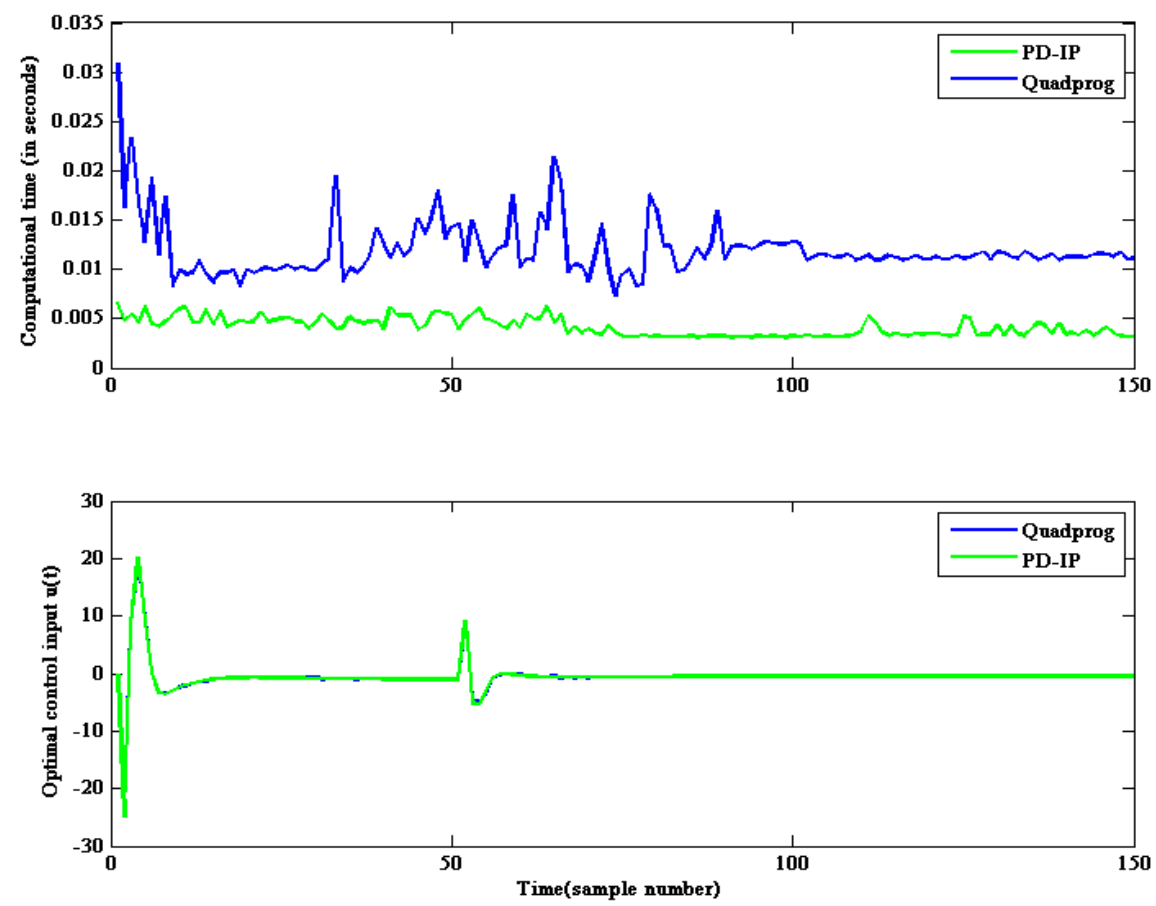

Figure 4. Plot of computational time comparison for $\mathrm{u}(\mathrm{t})$

\section{CONCLUSION}

In this paper, we presented a linear model predictive control of an undamped oscillator. The primal dual interior point method has been used to solve the QP optimal control problem arising in MPC. The logarithmic barrier merit function is used in such a way that it enables faster convergence of iterates while they remain strictly feasible. From the simulations; it is found that the proposed method is robust in the sense that it considerably rejects the output disturbances. For a given example, the proposed method was able to compute the optimal solution of the MPC problem approximately 3 times faster than that of using Quadprog without affecting the accuracy of $u_{t}$ computations.

\section{ACKNOWLEDGEMENTS}

We would like to thank our senior colleagues Vihang Naik and Deepak Ingole for their helpful technical discussions with us on the concept of MPC problem formulation.

\section{REFERENCES}

[1] C. Rao, S. J. Wright, and J. B. Rawlings, "Application of interior point methods to model predictive control", Journal of Optimization Theory and Applications, pp. 723-757, 1998.

[2] Carlos E. Garcia, D. M. Prett, and M. Morari, Model Predictive Control: theory and practice - a survey. Automatica, 25-335-348,1989.

[3] Liuping Wang, Model Predictive Control Design and Implementation Using MATLAB. SpringerVerlag London Limited, 2009.

[4] J. M. Maciejowski, Predictive control with constraints. Eaglewood Cliffs, NJ: Prentice Hall, 2002. 
[5] Yang Wang and Stephen Boyd, "Fast Model Predictive Control Using Online Optimization", IEEE Transactions on Control Systems Technology, Vol. 18, No. 2, March, 2010.

[6] A. G. Wills, W. P. Heath, "Interior-Point methods For Linear Model Predictive Control", In: Control, UK, 2004.

[7] Don Hush, Patrick Kelly, Clint Scovel, Ingo Steinwart, "QP Algorithms with Guarantied Accuracy and Run Time for Support Vector Machines”, Journal of Machine Learning Research 7, pp. 733-769, 2006.

[8] S. Mehrtotra, "On implementation of a primal-dual interior-point method", SIAM Journal on Optimization, 2(4), 575-601, (1992).

[9] Stephen Boyd and Lieven Vandenberghe, Convex Optimization, Cambridge University Press, 2009.

[10] Vihangkumar Naik, D. N. Sonwane, Deepak Ingole, Divyesh Ginoya and Neha Girme, "Design and Implementation of Interior-Point method Based Linear Model Predictive Controller", AIM/CCPE 2012, CCIS 296, Springer Berlin Heidelberg, pp. 255-261, 2013.

[11] Vihangkumar Naik, D. N. Sonawane, Deepak Ingole, L. Ginoya and Vedika Patki, "Design and Implementation of Proportional Integral Observer based Linear Model Predictive Controller", ACEEE Int. J. On Control System and Instrumentation, pp. 23-30, Vol. 4, No. 1, 2013.

[12] A. Bemporard, M. Morari, V. Dua, and E. N. Pistikopoulos, "The explicit linear quadratic regulator for constrained systems", Automatica, vol.38, no.1, pp. 3-20, Jan., 2002.

[13] J. B. Rawlings: Tutorial overview of model predictive control technology, IEEE Control Systems Magazine, 20:38-52, 2000.

[14] Y. Nesterov and A. Nemirovski, "Interior-Point polynomial methods in convex programming", Warrandale, PA: SIAM, 1994.

[15] K. M. Anstreicher and J. P. Vial, "On the convergence of an infeasible primal-dual interior-point method for convex programming”, Optim. Methods Softw., 3, pp. 273-283, 1994.

[16] A. G. Wills and W. P. Heath, "Barrier Function Based Model Predictive Control", Automatica, Vol. 40, No. 8, pp. 1415-1422, August, 2004.

[17] P. Armand, J. Gilbert, and S. Jan-Jegou, "A Feasible BFGS Interior Point Algorithm for Solving Convex Minimization Problems”, SIAM J. OPTIM, Vol. 11, No. 1, pp. 199-222, 2000.

\section{Authors}

Prashant Bansode received the B.E degree in Instrumentation Engineering from The University of Mumbai, in 2010 and the M.Tech degree in Instrumentation and Control Engineering from College of Engineering Pune, in 2013. His current research interests include convex optimization with applications to control, embedded system design, and implementation of numerical optimization algorithms using FPGA platforms.

Dr. D. N. Sonawane received the B.E degree in Instrumentation and Control Engineering from S.G.G.S College of Engineering, Nanded, in 1997. He received the M.E in Electronics and Telecommunication Engineering and the Ph.D degree in Engineering from College of Engineering Pune, in 2000 and 2012 respectively. He joined College of Engineering Pune as a lecturer in 1998, currently he is an Associate Professor with the Instrumentation and Control Department, College of Engineering Pune. He is the author of the book Introduction to Embedded System Design (ISTE WPLP, May, 2010). His research interests include Model Predictive Control, Quadratic Programming solver and their hardware implementation and acceleration using FPGA platforms. He is also involved in various research projects funded by different agencies of Govt. of India. He is a recipient of Uniken Innovation Award for the project "Sub Cutaneous Vein Detection System for Drug Delivery Assistance: an Embedded Open Source Approach", August, 2012, Followed by numerous National level awards. His paper titled "Design and Implementation of Interior-point Method based Linear Model Predictive Control" received best paper award at International Conference on Control, Communication and Power engineering, Bangalore, India, 2012.

Prashant Basargi received the B.E degree in Instrumentation Engineering from Shivaji University, in 2011 and the M.Tech degree in Instrumentation and Control Engineering from College of Engineering Pune, in 2013. His current research interests include Model Predictive Control, implementation of numerical optimization algorithms and Quadratic programming solvers using FPGA platforms. 